\title{
Follow-Up after Breast Cancer Diagnosis
}

\author{
Chair: Christoph Thomssen ${ }^{\mathrm{a}}$ \\ Participants: Ingo Diel ${ }^{b}$ Michael Gnant ${ }^{c}$ Ursula Goldmann-Posch ${ }^{d}$ \\ Christiane Göschke ${ }^{e}$ Renate Haidinger ${ }^{f}$ Wolfgang Jannig Nancy U. Lin ${ }^{\text {h }}$
}

aDepartment of Gynecology, Martin-Luther University Halle-Wittenberg, Halle an der Saale

${ }^{b}$ Schwerpunktpraxis für gynäkologische Onkologie, Praxisklinik am Rosengarten, Mannheim, Germany

'Department of Surgery, Comprehensive Cancer Center, Medical University of Vienna, Austria

${ }^{d}$ Mamazone and PONS-Foundation, Augsburg, Germany

${ }^{\mathrm{e} E u r o p a}$ Donna Austria, Vienna, Austria; ${ }^{\mathrm{f} B r u s t k r e b s ~ D e u t s c h l a n d ~ e . V ., ~ H o h e n b r u n n ~}$

9Department of Obstetrics and Gynecology, University UIm, Germany

hDepartment of Medicine, Harvard Medical School, Dana-Farber Cancer Institute, Boston, MA, USA

Follow-up after breast cancer is still an issue of debate. Modern diagnostic methods are more sensitive, and today many more effective therapeutic options are available. Thus, we think it is worthwhile to discuss the different opinions and approaches. The experts we asked come from Austria, Germany, Switzerland, and the USA.

\section{Question 1: The Course of Disease of Breast Cancer as well as the Chance of Cure Has Substantially Improved. Thus, also the Number of Patients Who Will Suffer from Recurrences During the First 5 Years after Diagnosis Has Been Reduced. Would You Still Consider a Regular Follow-Up Necessary and Where Would You See Its Future Goals?}

Ursula Goldmann-Posch: Breast cancer in women remains the most frequently occurring type of cancer and leading cause of cancer death. To improve survival rates, regular follow-up care is mandatory. I have never been a fan of indiscriminate procedures, but I strongly advocate follow-up care that is based upon the molecular principles specific to each tumor subgroup, and which respects the timeframes and locations of potential metastatic disease associated with this subgroup. Proactive rather than reactive behavior is of the utmost importance.

Christiane Göschke: To avoid neglecting possible early stages of breast cancer, which may concern as well the breast not primarily involved, I suggest follow-up every year or in even shorter intervals at the beginning.
Ingo Diel: Nowadays, $80 \%$ of all breast cancer patients can be cured and, thus, the minority of women would benefit from an intensive follow-up. However, I am convinced that we will increasingly be successful in treating and curing particularly patients with oligometastatic disease; and, of course, early detection of metastatic disease is an important prerequisite aiming at this goal.

Wolfgang Janni: I strongly believe that follow-up in the context of a surveillance program is needed for patients after breast cancer, even in cases of very good prognosis. Not only the early detection of recurrence, but many other aspects accompanying survivorship, such as counseling and guideline adherent long-term treatment may and should be part of the follow-up.

Michael Gnant: I consider regular and systematic follow-up extremely important. The purpose of this strategy is not only detection of relapses (we are struggling with proving that this is beneficial), but managing treatments and most importantly their side effects as well as assisting the patients in coping with the disease. The intensity of diagnostic measures should be risk-adapted.

Renate Haidinger: Follow-up care is very important, not only in reference to recurrences but also to side effects of treatments that were conducted or are still ongoing. 5 years of follow-up are not enough as more than half of recurrences in patients with hormone receptor (HR) positive disease occur after 5 years. It would be helpful to be able to differentiate more between high-risk and low-risk patients. Maybe subgroups could be defined who do not need the same surveillance intensity as high-risk patients.

\section{KARGER \\ Fax +497614520714 \\ Information@Karger.com}

www.karger.com (c) 2013 S. Karger GmbH, Freiburg

$1661-3791 / 13 / 0086-0457 \$ 38.00 / 0$

Accessible online at:

www.karger.com/brc
Prof. Dr. med. Christoph Thomssen

Klinik und Poliklinik für Gynäkologie

Martin-Luther-Universität Halle-Wittenberg

Ernst-Grube-Strasse 40

06097 Halle / Saale, Germany

christoph.thomssen@medizin.uni-halle.de 
Nancy Lin: I think that follow-up has different goals in different types of patients. For patients with estrogen receptor (ER) positive breast cancer, part of the goal is to query for adherence to endocrine therapy and to manage treatmentemergent side effects. For patients with triple-negative breast cancer, recurrences tend to occur early so for patients to have a connection with their oncologist especially in the first few years after diagnosis is still important.

\section{Question 2: Many Patients' Advocacy Groups and also Their Oncologists Favor a Scheduled 'Programmed' Surveillance, Including Regular Technical Examinations and Extensive Laboratory Tests Although Currently Available Data Definitely Do Not Support Such a Practice. Can You Explain this Inconsistent Situation?}

Christiane Göschke: After having gone through the ordeal of breast cancer treatment, patients wish to make sure that possible future troubles will be minimized by early detection.

Nancy Lin: The current randomized data and Cochrane analysis do not support regular bloodwork or body scans. Patients should still undergo regular breast imaging, unless they have had mastectomies. I think there is always a temptation to 'do more' and to hope that regular testing will reduce patient anxiety, and explaining the reasons not to do testing requires time and finesse on the part of the clinician.

Wolfgang Janni: In my view, surveillance is not only defined by imaging or lab tests. I absolutely acknowledge the mutual desire to potentially improve prognosis by early detection of recurrence, and the need for new scientific data, evaluating whether intensified surveillance could contribute to a better prognosis. Unfortunately, the only available randomized data are from the 1990s and therefore have reduced validity.

Michael Gnant: The scientific evidence demonstrating the lack of benefit of programmed follow-up and surveillance is essentially 20 years old and definitely outdated. When relapsing breast cancer is a condition for which not much can be done anyway, it does probably not make too much difference at what point in time a diagnosis of generalized disease is made. In contrast, I nowadays see patients surviving metastatic disease for 10 or 15 years, and the median survival time has greatly increased, for some subtypes even doubled and tripled.

Ursula Goldmann-Posch: For over a decade, patients and scientists of Mamazone and the PONS foundation have pointed out that the scientific basis for current breast cancer follow-up procedures is hopelessly outdated. Furthermore, due to a study design that is full of inadequacies concerning the early detection and early treatment of metastatic disease, the recently published follow-up study of Dr. Sven Bornhak and coworkers only increases the number of unanswered questions. Mamazone and the PONS foundation fight against follow-up care that is based upon a 'one size fits all' philosophy. We call for follow-up care to be effectively tailored to each molecular subgroup of breast cancer, and to be implemented when the risk of relapse for the respective group is highest.

Renate Haidinger: Relapses are what patients are afraid of and worry most about. Regular follow-up care is very important and I strongly agree that we need new data for this procedure. At the moment there is no evidence that an early detection of metastases with its consequent early therapy can really improve survival. Nowadays there are so many subtypes that can be defined with molecular testing, there could be a highly improved diagnostic potential and lots of new treatment options. Thus we need to know if we could and should change today's standards.

Ingo Diel: Of course, breast cancer patients and their advocacy groups - as we all - are interested in improving survival rates, particularly when metastasis did occur. Unfortunately, none of the randomized trials on follow-up has ever demonstrated a survival benefit by intensive surveillance when compared to less intensive clinical surveillance. Affected patients often argue that new treatment options in the metastatic situation have already shown better outcome. And reports on some (few) cases with long-term survival after treatment for oligometastatic disease increase their hope for cure. In these cases, single or few metastases were discovered by chance and consequently treated with curative intent. I think that the times will change. And we therapists should decide whether we want to form and design the future in terms of better surveillance instead of just quoting the old studies.

\section{Question 3: Could You Think of Patients with Certain Risk Criteria Who Would Potentially Benefit From an Intensified Follow-Up Strategy?}

Christiane Göschke: It is the doctor's job to define risk criteria and to give best practical advice. All breast cancer patients with special risk criteria will undoubtedly benefit from intensified follow-up.

Ursula Goldmann-Posch: If risk criteria were precisely defined using modern techniques, and if individualized followup programs were applied respective to the particularities of each risk group, I imagine that intensified follow-up care would make sense. By intensified I mean that modern diagnostic steps should be employed and that proper follow-up care should be given during those times when metastatic disease is most likely to reoccur. 
Renate Haidinger: The risk criteria for each individual patient should be defined although we do not have strong data confirming this procedure. Maybe bad responders in neoadjuvant treatments or node-positive patients might profit. Doctors should discuss this option - although again, we do not have new data for this.

Nancy Lin: I don't think there are any patient groups that we know of that could benefit. In subset analyses of the randomized trials, even node-positive patients did not gain a survival benefit with intensified surveillance.

Ingo Diel: Of course, these are at first those patients who have an increased risk of metastasis due to the aggressive biology of their tumor. Included are patients with already infiltrated lymphatic vessels and lymph nodes. Also patients with fast growing tumors (G III, Ki $67>20 \%$, etc.) would be potential candidates for a more intensive follow-up.

Wolfgang Janni: We currently do not have sufficient data to select patients for intensified surveillance based on prognostic or predictive factors. Patients with high-risk disease might benefit most, but potentially also patients with long-term risk, such as patients with hormone responsive disease might benefit. This should also be part of future scientific evaluations.

Michael Gnant: This relates to the response pattern of the individual breast cancer subtype: For triple-negative breast cancer with its early relapse peak, I usually re-call my patients every 3 months - for luminal A patients, once a year but lifelong surveillance might be the optimal approach.

\section{Question 4: What Should Currently Be the Role of Modern Techniques, such as Positrone Emission Tomography (PET, PET-CT), Tumor Markers, Detection of Circulating Tumor Cells etc. with Regard to Surveillance after Diagnosis of Breast Cancer?}

Nancy Lin: I personally do not perform any of these tests on a routine basis in asymptomatic patients. I would have a low threshold to order selective imaging studies or other tests based on patient symptoms of concern.

Wolfgang Janni: Probably both the low sensitivity of conventional methods, such as chest $\mathrm{x}$-ray, and the limited spectrum of intervention options might have contributed to the negative results of the early surveillance studies of the 1990s. In my view, new studies should include more sensitive methods to detect minimal residual disease.

Michael Gnant: These methods have ups and downs: we do mammography and blood tests regularly, with less and less tumor markers. CT scans are used earlier in the course of follow-up and in high-risk patients. I do not regularly recommend PET, PET-CT, or CTC.

Ursula Goldmann-Posch: The fact that not a single pathologist, laboratory doctor, radiologist, nuclear medicine specialist, surgeon, or radiotherapist has been asked to contribute to this expert discussion demonstrates the need for more interdisciplinary cooperation when it comes to breast cancer follow-up. It is a waste of time to go over old-fashioned followup care trials again and again. We need new ideas, and we must employ modern tools, up-to-date knowledge of serological diagnostic oncology, and whole-body imaging in follow-up care. The best diagnostic tools, however, are useless if physicians are unwilling or unprepared to treat a limited asymptomatic local metastatic disease with modern interventional radiology or minimally invasive surgery, in combination with targeted systemic therapy.

Christiane Göschke: This is a question to be answered by experts. The advocate's point of view suggests that modern techniques should be used to make sure that no metastases have spread out. On the other hand, too many and certainly for the patient frightening technical examinations should be avoided.

Renate Haidinger: We do not have enough data on the benefit of modern techniques, thus a regular use does not make sense but might be effective for some patients.

Ingo Diel: Tumor markers could play an important role, although sensitivity may be far too low. But determination of the markers is cheap and easy to carry out. At present, classical staging methods (bone scan, CT and MRI) should be considered only in cases with very high risk. PET/CT and wholebody MRI, or the detection of circulating tumor cells are very promising techniques, but need to be sufficiently tested in prospective studies. On the other hand, these methods are complicated and expensive.

\section{Question 5: More Than Half of All Recurrences Occur Beyond the Fifth Year After Breast Cancer Diagnosis. Are Regular Follow-Up Visits in the First 5 Years Really Helpful and Is It Correct to Stop the Recommendation for Follow-Up Programs at this Time Point?}

Michael Gnant: Definitely not. Follow-up has to be lifelong, particularly for luminal cancer types.

Christiane Göschke: Follow-up in the first 5 years after diagnosis should be state of the art for all patients. However, the use of technology should be adapted to the special case. 
Even if in the following period more relapses will occur, those within the first 5 years should not be neglected. Another important argument is that a trustful patient/doctor relation can be formed during this time.

Ursula Goldmann-Posch: The currently used guidelines along with the so-called 5-year probation period are based on an erroneous 'one size fits all' dogma. The fallacy of this belief is illustrated by the frequent luminal subtypes of breast cancer that metastasize late. Why the 2011 St. Gallen International Breast Cancer Conference adopted adjuvant treatment recommendations appropriate to breast cancer subtypes, but yet was unable to adjust its follow-up care recommendations to these subtypes remains a mystery to me. Follow-up care must consist of caring for every single breast cancer patient during the time in which it is most effective, and with those strategies best adapted to her tumor's subtype.

Ingo Diel: We know that a different biology of breast cancer leads to different frequencies of recurrences in the course of the disease. When designing studies on the impact of follow-up, this has to be taken into account by choosing distinct schedules and technical methods of surveillance. Aimed at early detection of oligometastatic, potentially curable disease, clear concepts for the type of therapy have to be established. The increasing numbers of my own patients with long-time survival despite metastatic disease support the hope of cure.

Wolfgang Janni: The longitudinal probability of recurrence greatly depends on tumor biology and maybe in future, the duration of surveillance may be guided by the subtype of the primary disease.

Renate Haidinger: No. We all know that there are more than $50 \%$ of the relapses in HR positive breast cancer after 5 years. Long-term side effects also might occur after this 5 -year period. Therefore after 5 years, regular annual followup care should be standard of care.

Nancy Lin: This is true for patients with ER-positive breast cancer. I personally follow such patients indefinitely. My own practice is to see patients approximately every 6 months (or more often if there are intervening issues) so long as I am prescribing them a medication (e.g. tamoxifen or an aromatase inhibitor). Once the patient has completed her medications, I reduce the frequency of follow-up to once yearly, at the time of yearly breast imaging (if applicable). I think that the regular follow-up visits beyond year 5 are helpful to keep a connection and so patients feel comfortable calling me if new symptoms arise between visits and still feel a tie to my practice. For patients with triple-negative breast cancer, in whom the risk of recurrence after 5 years is very low, I offer the option of suspending follow-up with me and returning to their primary care physician. Some of the patients would prefer to continue to have their annual mammograms at our comprehensive cancer center, and I do give patients that option. In that case, they see a nurse practitioner for a breast examination and review of mammogram results once yearly as well.

\section{Participants}

Prof. Dr. med. Ingo Diel

Schwerpunktpraxis für Gynäkologische Onkologie Praxisklinik am Rosengarten

Augustaanlage 7-11, 68165 Mannheim, Germany

idiel@spgo-mannheim.de

Professor Michael Gnant, M.D., FACS

Department of Surgery and Breast Health Center Vienna

Comprehensive Cancer Center Vienna

Medical University of Vienna

1090 Wien, Währinger Guertel 18-20, Austria

michael.gnant@meduniwien.ac.at

Ursula Goldmann-Posch, patients' advocate mamazone - Frauen und Forschung gegen Brustkrebs e. V. Postfach 310220, 86063 Augsburg, Germany goldmann.posch@t-online.de

Christiane Göschke, patients' advocate

Europa Donna Austria

c/o Grichemonde, Goldschmiedgasse 2/6/5, 1010 Vienna, Austria info@europadonna.at

Renate Haidinger, patients' advocate Brustkrebs Deutschland e.V. Lise-Meitner-Str. 7, 85662 Hohenbrunn, Germany renate.haidinger@web.de

Prof. Dr. med. Wolfgang Janni Frauenklinik

Universitätsklinikum Ulm Prittwitzstr. 43, 89075 Ulm, Germany

wolfgang.janni@uniklinik-ulm.de

Prof. Nancy U. Lin, M.D.

Department of Medicine, Harvard Medical School

Dana-Farber Cancer Institute

450 Brookline Avenue

Boston, MA 02215, USA

Nancy_Lin@dfci.harvard.edu 